\title{
Informal use of PrEP among MSM STI clinic visitors: preliminary results of the InPrEP study
}

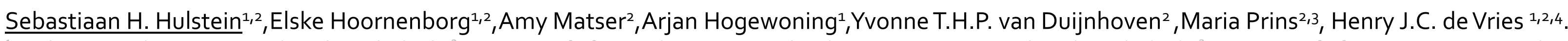

bhulstein@ggd.amsterdam.nl

\section{Background}

Pre-exposure prophylaxis (PrEP) is highly effective to prevent HIV among men who have sex with men (MSM). In the Netherlands PrEP is not yet implemented and reimbursed. An unknown number of MSM use "informal" PrEP, i.e. self-obtained PrEP without medical supervision and prescription. In the InPrEP study, we assessed informal PrEP use among Amsterdam STI clinic visitors and offered care.

\section{Objectives}

- To evaluate PrEP use, eligibility and preferred dosing regimens among informal PrEP users

- To evaluate STI, HIV and hepatitis C diagnoses among informal PrEP users in comparison to non-PrEP using, HIV-negative MSM visiting the STI clinic.

\section{Methods}

- Inclusion: HIV-negative MSM STI clinic visitors who either:

1. had self-obtained PrEP without prescription

2. intent to start using (self-obtained) PrEP and not participating in a PrEP study.

- Care: Quarterly testing of STI (chlamydia, gonorrhea, syphilis, hepatitis $B$ if unvaccinated, hepatitis $C$ and HIV), renal function and counseling on PrEP-related topics. If necessary, PrEP prescriptions were provided. Costs for medication can not be reimbursed via health insurance.

- Measurements: observation of adverse events; questionnaires on subjects including PrEP use and dosing regimens.

- Description of 1. eligibility for PrEP use and dosing regimen among InPrEP participants and 2. comparison to between InPrEP participants and non-PrEP-using MSM.

\section{Results}

Between October 2017 and February 2018, informal PrEP use in the past 3 months was reported by $248 / 3326$ (7.5\%) HIV-negative MSM. In total 145 (intended and active) informal PrEP users were included (see Figure 1). $82.4 \%$ of participants were eligible to use PrEP according to Dutch PrEP guidelines (see table 1). Preferred dosing regimens were: $48.2 \%$ daily, $35.2 \%$ event-driven, $7.4 \%$ both and $9.3 \%$ other non-evidence based regimens. STI prevalence is stated in table 2. Among informal PrEP users two hepatitis C, but no HIV and hepatitis B infections were observed.

Table 1. Eligibility criteria for using PrEP according to the Dutch PrEP guidelines.

MSM or transgender who has had on or more of the following in the preceding 6 months

\begin{tabular}{|l|l|}
\hline Rectal STI & $30.4 \%$ \\
\hline Course of PEP & $27.5 \%$ \\
\hline Sex partner with unknown HIV status & $68.7 \%$ \\
\hline HIV positive sex partner (detectable) & $4.9 \%$ \\
\hline Any & $82.4 \%$ \\
\hline
\end{tabular}

Table 2. STI prevalence for informal PrEP users (at enrolment) and non-

PrEP using MSM.

\begin{tabular}{ll|l|l|l|l|} 
Bacterial STI & $\begin{array}{l}\text { InPrEP } \\
\text { participants } \\
\text { N=145 }\end{array}$ & $\begin{array}{l}\text { Non-PrEP } \\
\text { using MSM } \\
\mathrm{N}=2624\end{array}$ & $\begin{array}{l}\text { PrEP using } \\
\text { MSM, not } \\
\text { enrolled } \\
\mathrm{N}=103\end{array}$ & $\begin{array}{l}\text { "Other } \\
\text { studies" } \\
\text { PrEP use } \\
\mathrm{N}=389\end{array}$ & P value $\left(\chi^{2}\right)$ \\
\hline Chlamydia & $14(9.7 \%)$ & $249(9.5 \%)$ & $15(14.6 \%)$ & $23(5.9 \%)$ & 0.031 \\
\hline Gonorrhea & $22(15.2 \%)$ & $331(12.6 \%)$ & $23(22.3 \%)$ & $22(15.2 \%)$ & 0.025 \\
\hline Syphilis & $4(2.8 \%)$ & $58(2.2 \%)$ & $8(7.8 \%)$ & $10(2.6 \%)$ & 0.005
\end{tabular}

\section{HIV-negative MSM STI Clinic visitors between October 2017 and February 2018 $\mathrm{N}=3326$}

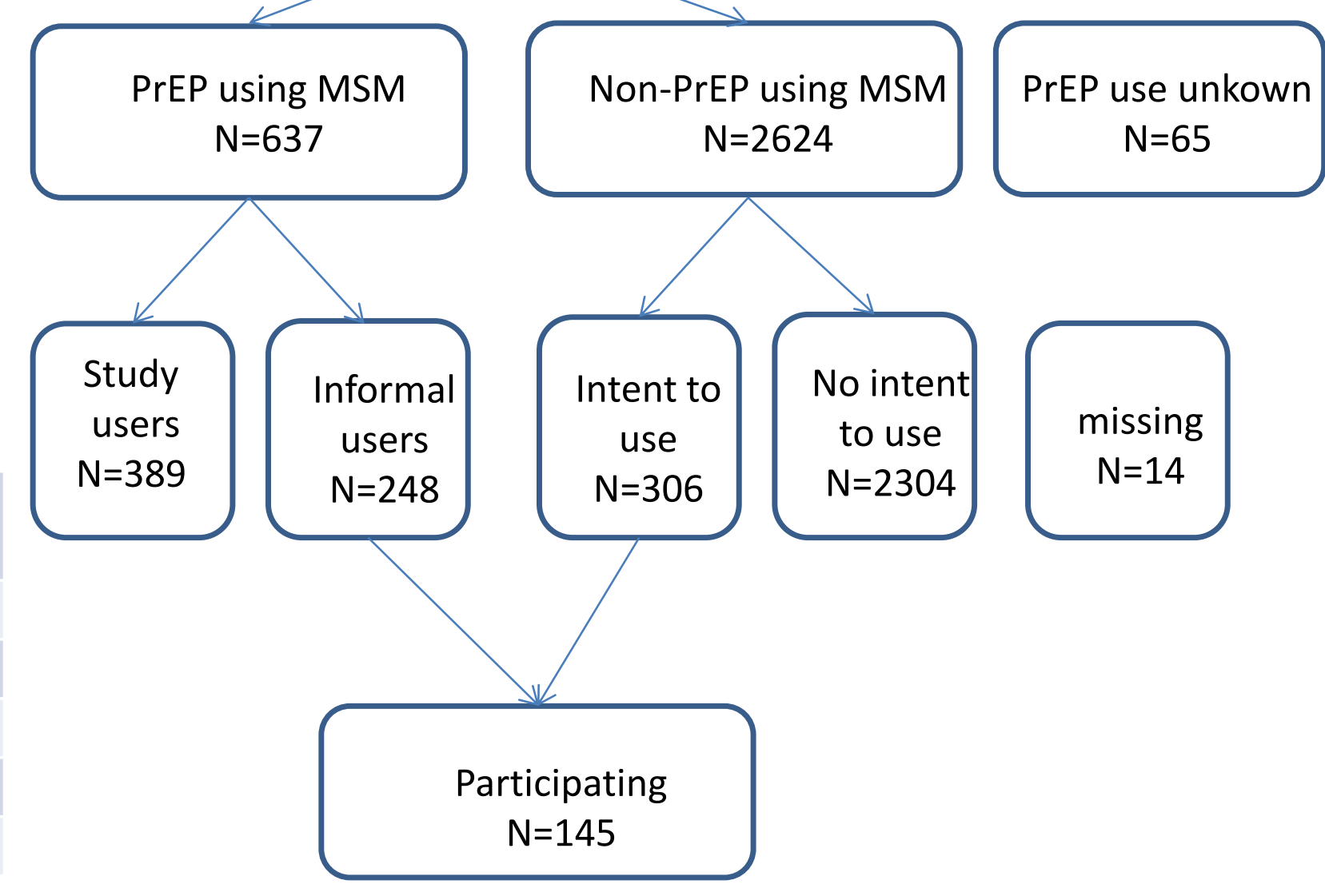

Figure 1. Flow chart of InPrEP study participants.

Study users are PrEP-using MSM who obtain PrEP from participation in another PrEP study.

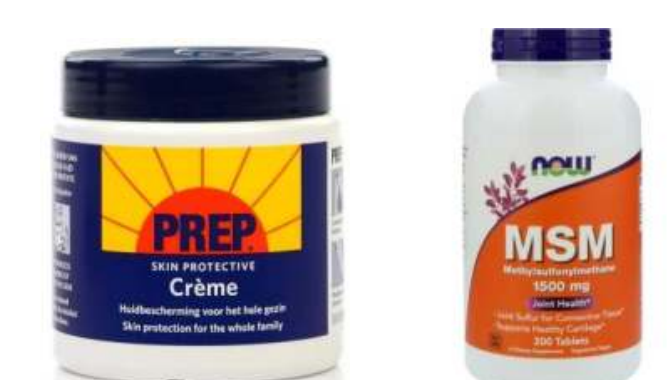

- InPrEP is an observational cohort of MSM who use self-obtained PrEP without reimbursement of medication costs.

- Informal PrEP use occurs among about $8 \%$ of HIV-negative MSM attending the Amsterdam STI clinic.

- Various dosing regimens are used, showing that both daily and event-driven regimens need to be offered to potential users to meet their needs.

- We found similar STI rates between informal PrEP using and non-PrEP using MSM, but higher rates among PrEP users, not participating in any study, which warrants extra attention. 